\title{
FACE RECOGNITION USING GAUSSIAN MIXTURE MODEL \& ARTIFICIAL NEURAL NETWORK
}

\author{
Jatinder Sharma ${ }^{1}$, Rishav Dewan ${ }^{2}$ \\ ${ }^{1}$ Bhai Gurdas Institute of Engg \& Technology, Sangrur \\ sharma.jatinder70@gmail.com \\ ${ }^{2}$ Bhai Gurdas Institute of Engg \& Technology, Sangrur \\ rishavdewan@gmail.com
}

\begin{abstract}
Face recognition is a non-contact and friendly biometric identification technology. It has broad application prospects in the military, public security and economic security. In this work, we also consider illumination variable database. The images have taken from far distance and do not consider the close view face of the individual as in most of the face databases, clear face view has been considered. In this first we located face as region of interest and then LBP and LPQ descriptors are used which is illuminance invariant in nature. After this GMM has been used to reduce feature set by taking negative log-likelihood from each $L B P$ and $L P Q$ descripted image histograms. After this ANN consumes stayed used for organization purposes. The investigational consequencesshow excellent correctness rates in overall testing of input data.
\end{abstract}

Keywords: Illumination invariant, face recognition, LBP, LPQs, GMM,ANN

\section{INTRODUCTION}

Face recognition is an interestingand challenging problem and impacts important application in many area such as identification for law enforcement, authentication for banking and security system acess and personal identification among other. Facial expression is one of the most powerful natural and immediate means for human beings to communicate their emotion and intensions. Although, over the past years, many different techniques to recognize faces across illumination changes have been proposed, but they still have drawbacks. The qualities of these systems are that they do not require any lighting assumption nor do they need any training. In this work, we have tried to combine the spatial and frequency domain features of illumination invariant images by considering local binary pattern and local phase quantization.

\section{LOCAL BINARY PATTERN:-}

The LBP operator was initially planned for texture explanation. The operator allocates a tag to each pixel of an appearance by thresholding the $3 \times 3$-neighborhood of each pixel with the midpoint pixel value and considering the outcome as a binary number. It is more accurate.It also describe the texture and shape of a digital image. The main advantage of LBP for large image is divided so that many different type of humanexpression can be easily recognised.

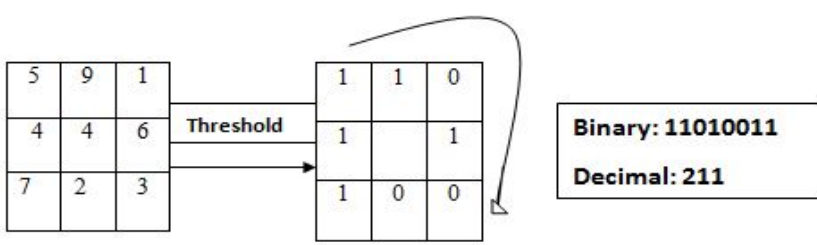

Fig 1:- Local binary pattern Method
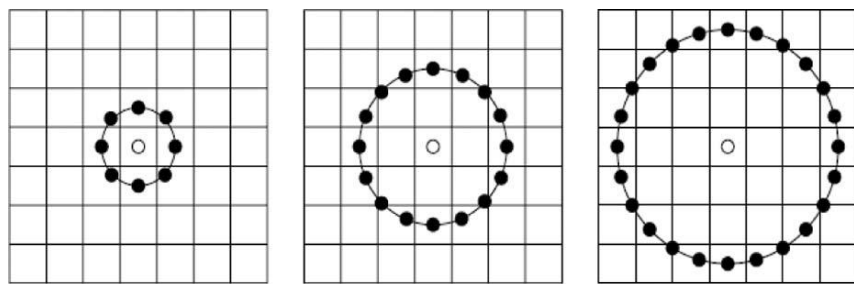

Fig 2:- Extended Local binary pattern Method and circular $(8,1),(16,2),(24,3)$ neighbourhood

\section{FORMULAS \& CALCULATIONS USED}

For calculateFace recognition using GMM Model we used this given formula which is given below

$\lambda=\left(\mathrm{w}_{\mathrm{i}}, \mu_{\mathrm{i}}, \Sigma_{\mathrm{i}}\right), \quad \mathrm{i}=1,2,3, \ldots, \mathrm{M}$. 


\section{PROPOSED SYSTEM}

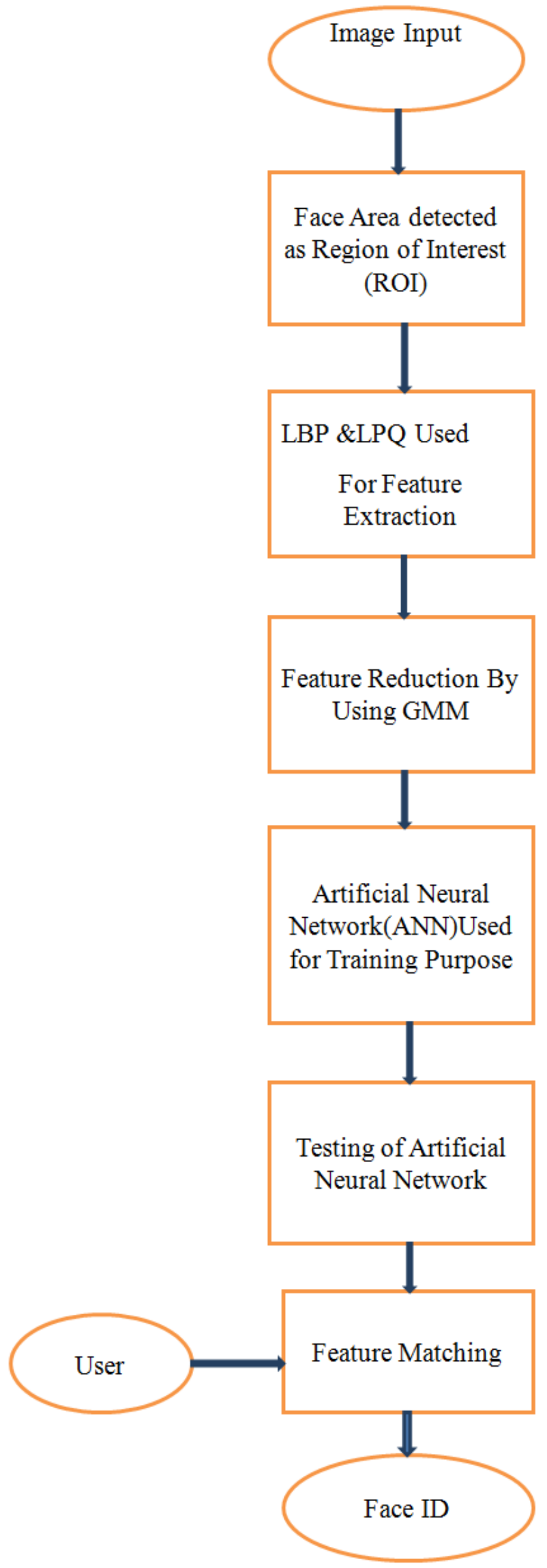

\section{GAUSSIAN MIXTURE MODEL:-}

- $\quad$ GMM Model is Used for reducing the feature space of the LBP and LPQ histogram matrices

- $\quad$ Firstly created Gaussian mixture models from LBP and LPQ geographies and then $\mathrm{N} \log 1$ (Negative log likelihood) is used as a feature for complete one network LBP or LPQ matrix.

- GMM always gives single value of each data set which is used by Artificial neural network.

\begin{tabular}{|c|c|c|c|c|c|c|}
\hline & $\begin{array}{l}\text { Log } \\
\text { Likelihood } \\
\text { of GMM } \\
\text { with LBP ch 1 }\end{array}$ & $\begin{array}{l}\text { Log } \\
\text { Likelihood } \\
\text { of GMM } \\
\text { with LBP ch } 2\end{array}$ & \begin{tabular}{|l|} 
Log \\
Likelihood \\
of GMM \\
with LBP ch 3
\end{tabular} & $\begin{array}{l}\text { Log } \\
\text { Likelihood } \\
\text { of GMM } \\
3 \text { with LPQ ch } 1\end{array}$ & $\begin{array}{l}\text { Log } \\
\text { Likelihood } \\
\text { of GMM } \\
1 \text { with LPQ ch } 2\end{array}$ & $\begin{array}{l}\text { Log } \\
\text { Likelihood } \\
\text { of GMM } \\
2 \text { with LPQ ch } 3\end{array}$ \\
\hline 1 & $9.0462 e+03$ & $7.6708 e+03$ & $7.7783 e+03$ & $8.4250 \mathrm{e}+03$ & $7.9197 e+03$ & $7.9789 e+03$ \\
\hline 2 & $8.7202 e+03$ & $8.0813 e+03$ & $7.7030 \mathrm{e}+03$ & $8.1641 e+03$ & $7.9145 e+03$ & $7.9166 e+03$ \\
\hline 3 & $9.0007 e+03$ & $7.7142 e+03$ & $7.7258 \mathrm{e}+03$ & $8.3274 e+03$ & $8.0195 e+03$ & $8.0433 e+0$ \\
\hline 4 & $9.0292 e+03$ & $7.6761 e+03$ & $8.0641 e+03$ & $8.4287 e+03$ & $7.9672 e+03$ & $8.0438 e+03$ \\
\hline 5 & $9.0697 e+03$ & $7.8026 e+03$ & $7.8688 e+03$ & $8.4705 e+03$ & $7.9846 e+03$ & $8.0931 \mathrm{e}+03$ \\
\hline 6 & $8.9349 e+03$ & $7.8573 e+03$ & $7.7194 e+03$ & $8.4461 e+03$ & $7.9612 e+03$ & $8.0377 e+03$ \\
\hline 7 & $8.6845 e+03$ & $8.0173 e+03$ & $7.7557 e+03$ & $8.1530 e+03$ & $7.9147 e+03$ & $7.8347 e+03$ \\
\hline 8 & $8.7411 e+03$ & $7.9191 e+03$ & $7.7435 e+03$ & $8.0803 e+03$ & $7.9661 \mathrm{e}+03$ & $7.9891 e+03$ \\
\hline 9 & $8.7309 e+03$ & $8.0533 e+03$ & $7.8436 e+03$ & $8.1083 e+03$ & $7.8932 e+03$ & $7.9985 e+03$ \\
\hline 10 & $8.7056 e+03$ & $7.9787 e+03$ & $7.6494 e+03$ & $8.0971 e+03$ & $7.9445 e+03$ & $7.9713 e+03$ \\
\hline 11 & $8.7304 e+03$ & $7.6937 e+03$ & $7.4415 e+03$ & $8.1120 e+03$ & $7.8771 e+03$ & $7.8246 e+03$ \\
\hline 12 & $9.0365 e+03$ & $7.7401 e+03$ & $7.9381 e+03$ & $8.5301 e+03$ & $7.9787 e+03$ & $8.0997 e+03$ \\
\hline
\end{tabular}

Fig 3:- By Using GMM Model Negative log Likelihood values fed in ANN Network

\section{(A) Feature Space Reduction Using Gaussian}

\section{Mixtures}

Features of each sub-block, which are extracted by two different descriptors (LBP and LPQ), are fed into Gaussian mixture modeling. In this step, histograms of both descriptor outputs are fed individually according to color space used in the algorithm. As RGB images are first converted to Lab color space, each color component has been put through both the descriptors separately and then their histograms are stored into a matrix. After GMM modeling negative loglikelihood value has been chosen for each color space which results in total six features from each input image. These features are then fed into artificial neural network for training and testing of images.

\section{ARTIFICIAL NEURALNETWORK}

ANN means "artificial neural network".It is related to brain structure.Basically it is a interconnection of various types of nodes .For example : large amount of neurons present in the brain. The next layer may turn in make it independent computations and pass on the result to yet another. In human mind various types of neurons are interconnect with each other and definitely they generate the signal in human mind.The benefit of ANN is that we have to trained only one time and after that we have testing multiple time 


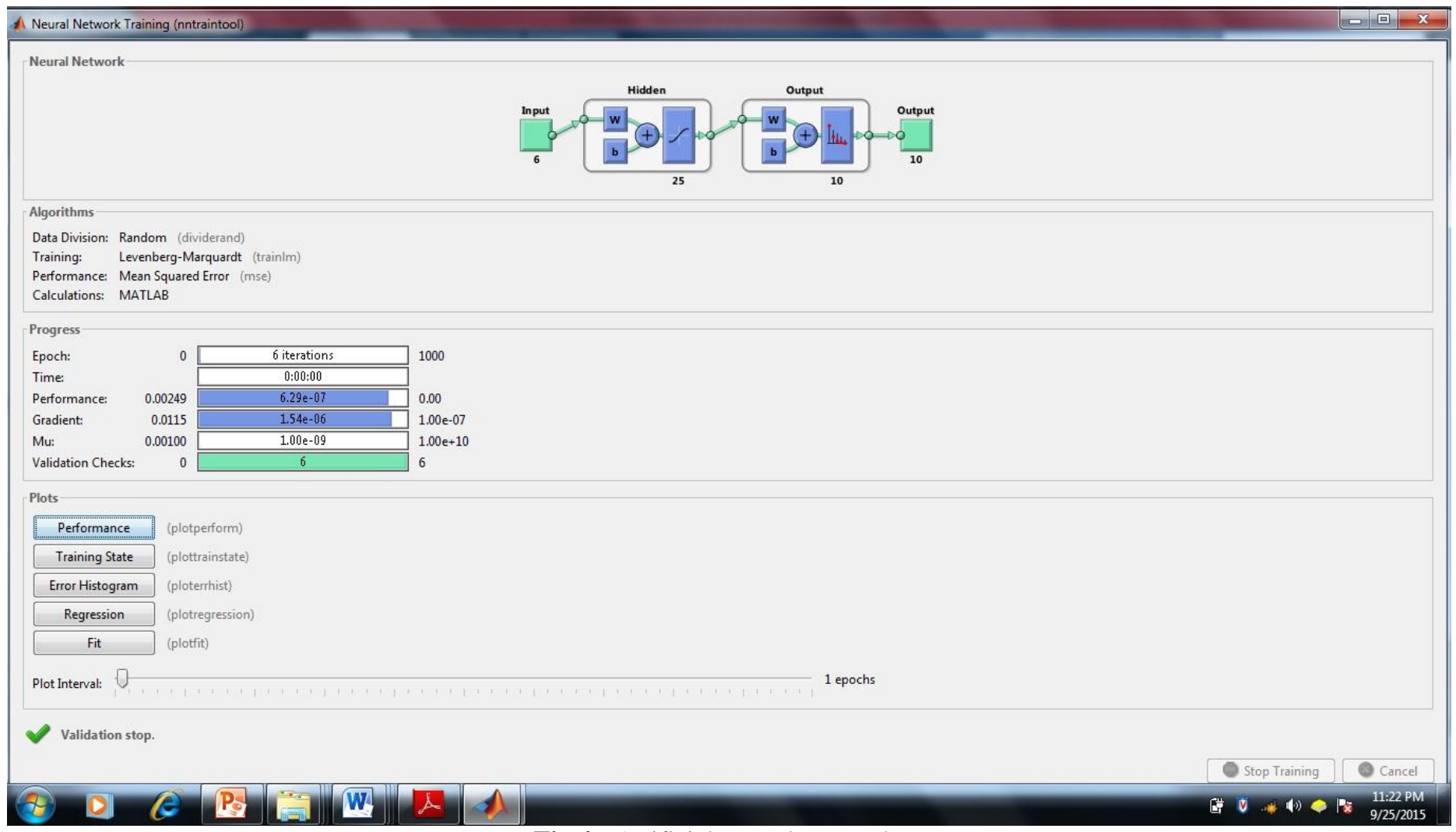

Fig 4:- Artificial Neural Network

ANN Work as Sequential and Brain work as parallel.In this

Levenberg-Marquardt Algorithm is used.

\section{FLOW OF ALGORITHM}

(A) Back Propagation Algorithm

Fundamental steps of Algorithm:-

1) Initialization of weights

2) Feed Forward

3) Back Propagation of errors

4) Updating of weight and biases 


\section{BACK PROPAGATION ALGORITHM}

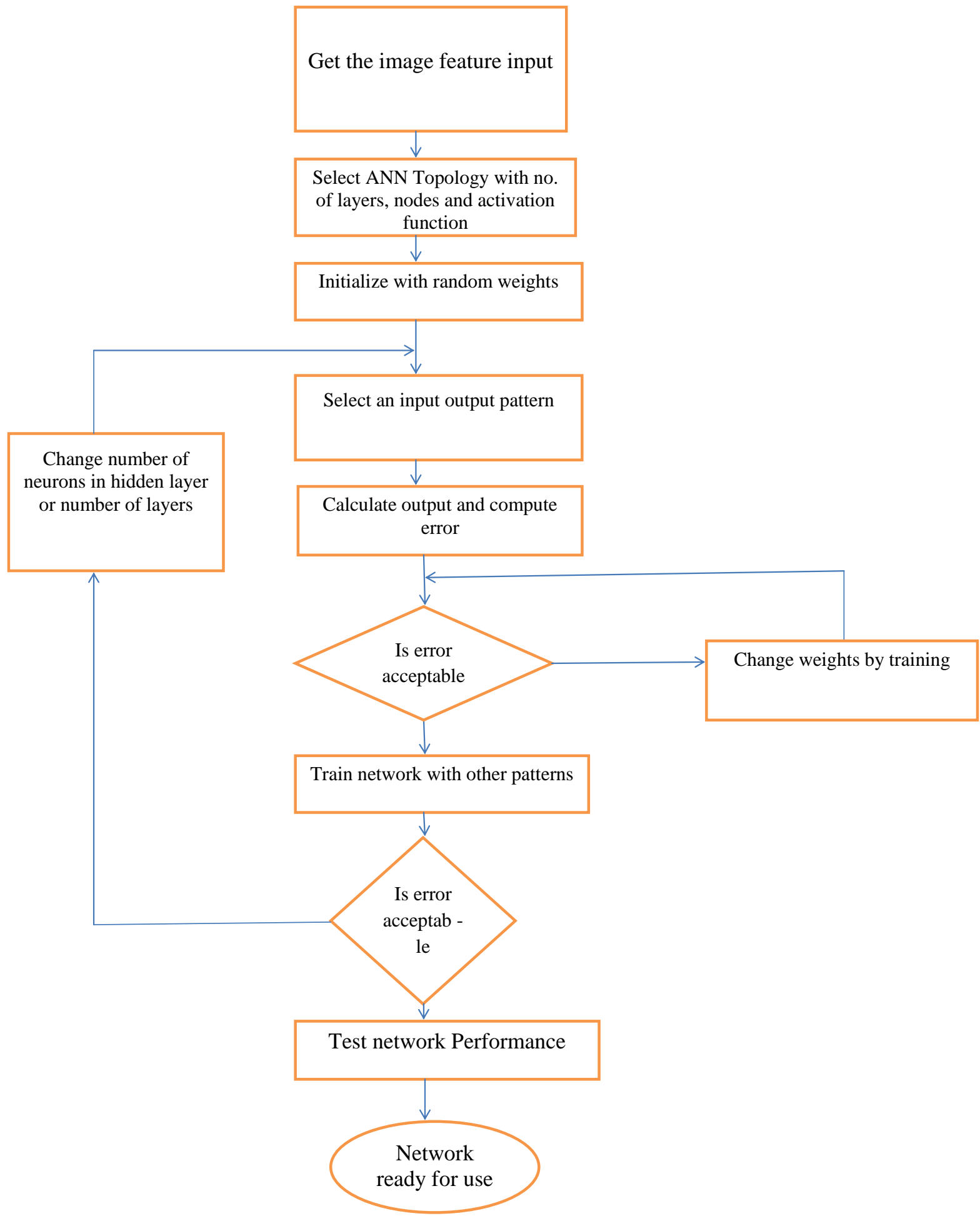

Fig 5 :- Back Propagation Algorithm

\section{RESULT ANALYSIS}

Experimental results has been carried out on a database of ten persons, in which five images are chosen in normal lightning conditions and five images are chosen on dim light. After getting the histograms of LBP and LPQ feature set, they are reduced to a vector of length six data entries by using Gaussian mixture models. After this ANN has been trained and tested. Performance of the ANN classifier has been checked by confusion matrix, sensitivity and specificity values. The confusion matrix of ANN testing output has been given below 


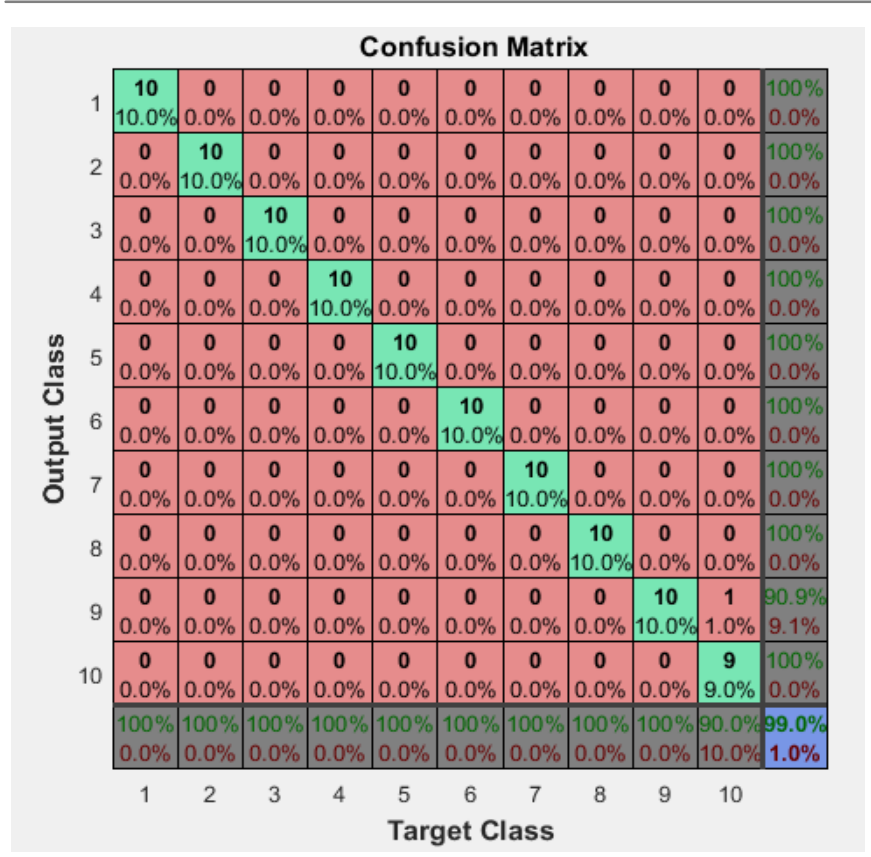

\begin{tabular}{|c|c|c|}
\hline \multirow{2}{*}{$\begin{array}{c}\text { Actual } \\
\text { Class }\end{array}$} & \multicolumn{2}{|c|}{ Predicted Class } \\
\cline { 2 - 3 } & Yes & No \\
\hline Yes & TP & FN \\
\hline No & FP & TN \\
\hline
\end{tabular}

Fig 6:- Overall Confusion Matrix

Table (a):- Confusion Matrix for Two class classifier

\begin{tabular}{|c|c|c|c|c|c|c|c|}
\hline $\begin{array}{c}\text { Database } \\
\text { individual }\end{array}$ & $\begin{array}{c}\text { True } \\
\text { Positive }\end{array}$ & $\begin{array}{c}\text { False } \\
\text { Negative }\end{array}$ & $\begin{array}{c}\text { True } \\
\text { Negative }\end{array}$ & $\begin{array}{c}\text { False } \\
\text { positive }\end{array}$ & Sensitivity & Specificity & Accuracy \\
\hline Person 1 & 10 & 0 & 10 & 0 & $100 \%$ & $100 \%$ & $100 \%$ \\
\hline Person 2 & 10 & 0 & 10 & 0 & $100 \%$ & $100 \%$ & $100 \%$ \\
\hline Person 3 & 10 & 0 & 10 & 0 & $100 \%$ & $100 \%$ & $100 \%$ \\
\hline Person 4 & 10 & 0 & 10 & 0 & $100 \%$ & $100 \%$ & $100 \%$ \\
\hline Person 5 & 10 & 0 & 10 & 0 & $100 \%$ & $100 \%$ & $100 \%$ \\
\hline Person 6 & 10 & 0 & 10 & 0 & $100 \%$ & $100 \%$ & $100 \%$ \\
\hline Person 7 & 10 & 0 & 10 & 0 & $100 \%$ & $100 \%$ & $100 \%$ \\
\hline Person 8 & 10 & 0 & 10 & 0 & $100 \%$ & $100 \%$ & $100 \%$ \\
\hline Person 9 & 10 & 0 & 10 & 0 & $90.9 \%$ & $100 \%$ & $95.45 \%$ \\
\hline Person 10 & 9 & 0 & 9 & 1 & $100 \%$ & $90 \%$ & $95 \%$ \\
\hline
\end{tabular}

Table (b):- Showing different parameters for evaluating performance of the algorithm

\begin{tabular}{|l|l|}
\hline Overall recognition rate using old method(SoodehNikan et al.) \\
\hline Database used & Recognition rate \\
\hline Accuracy Percentage of Yale B database & $98.30 \%$ \\
\hline Accuracy Percentage of AR database & $99 \%$ \\
\hline Accuracy Percentage of Multi-PIE database & \\
Session 2 & $97.54 \%$ \\
Session3 & $96 \%$ \\
Session4 & $98.7 \%$ \\
\hline Accuracy Percentage of First Experiment of Face & $32.62 \%$ \\
identification algorithm on the FRGC2.0.4 Database & \\
\hline Accuracy Percentage of Second experiment of & $23.3 \%$ \\
FRGC2.0.4 Database & \\
Recognition Accuracy & \\
\hline
\end{tabular}




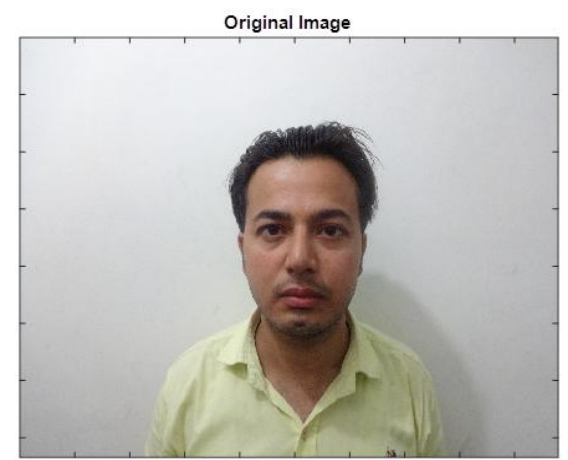

(a) Input image

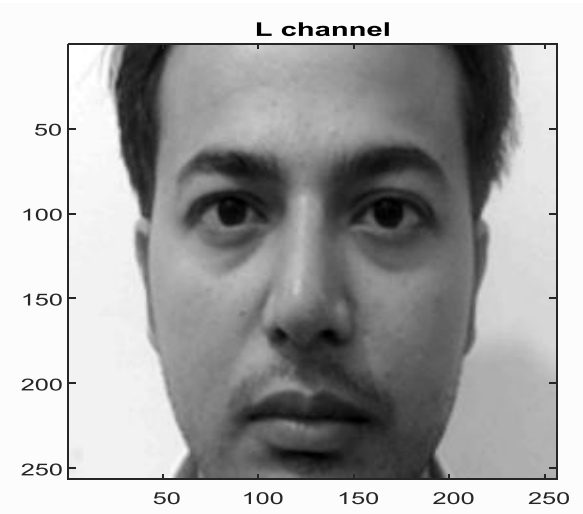

Figure7 (a) L channel of face localized image (b)

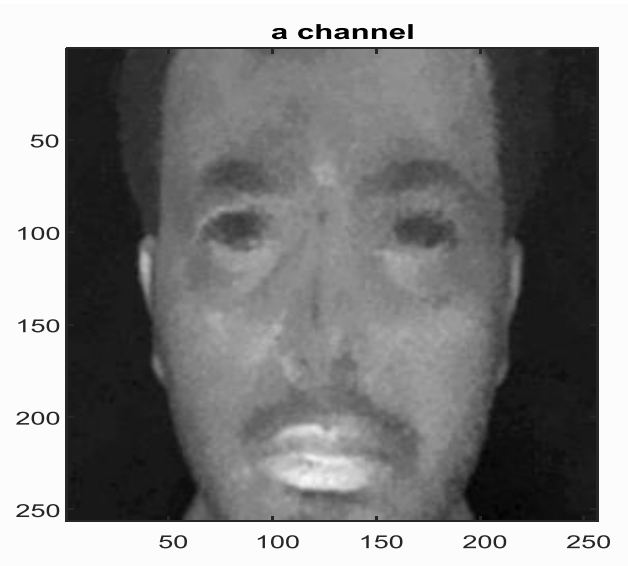

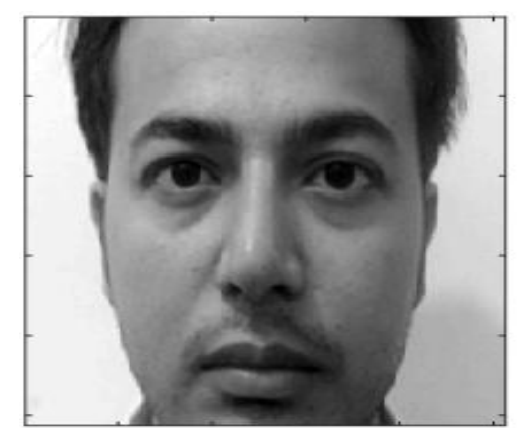

(b) Face area detected as region of interest

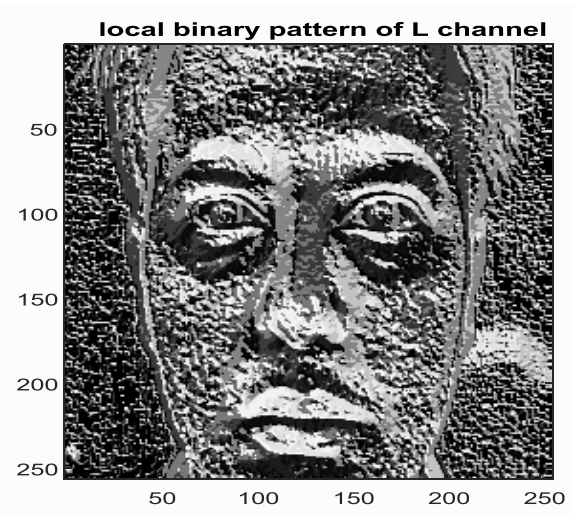

(b) LBP of L channel of face localized image

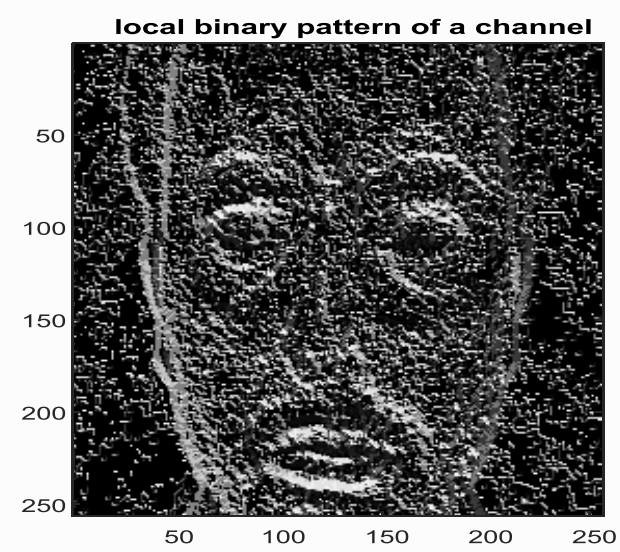

Figure8: (a) $a^{*}$ channel of face localized image (b) LBP of a* channel of face localized image
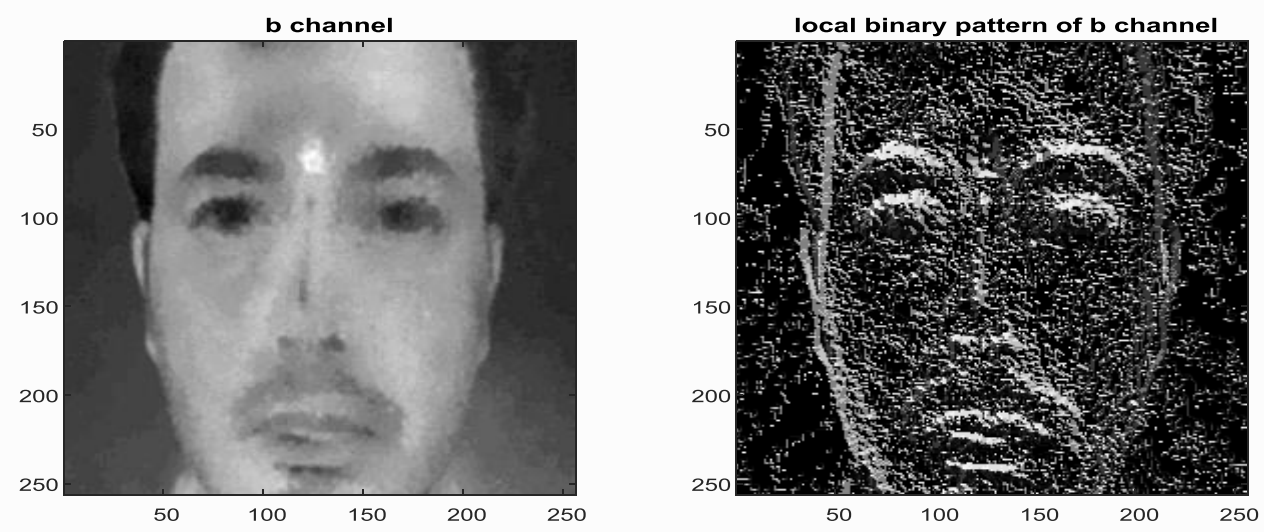

Figure 9 (a) b* channel of face localized image (b) LPQ of $b^{*}$ channel of face localized image 


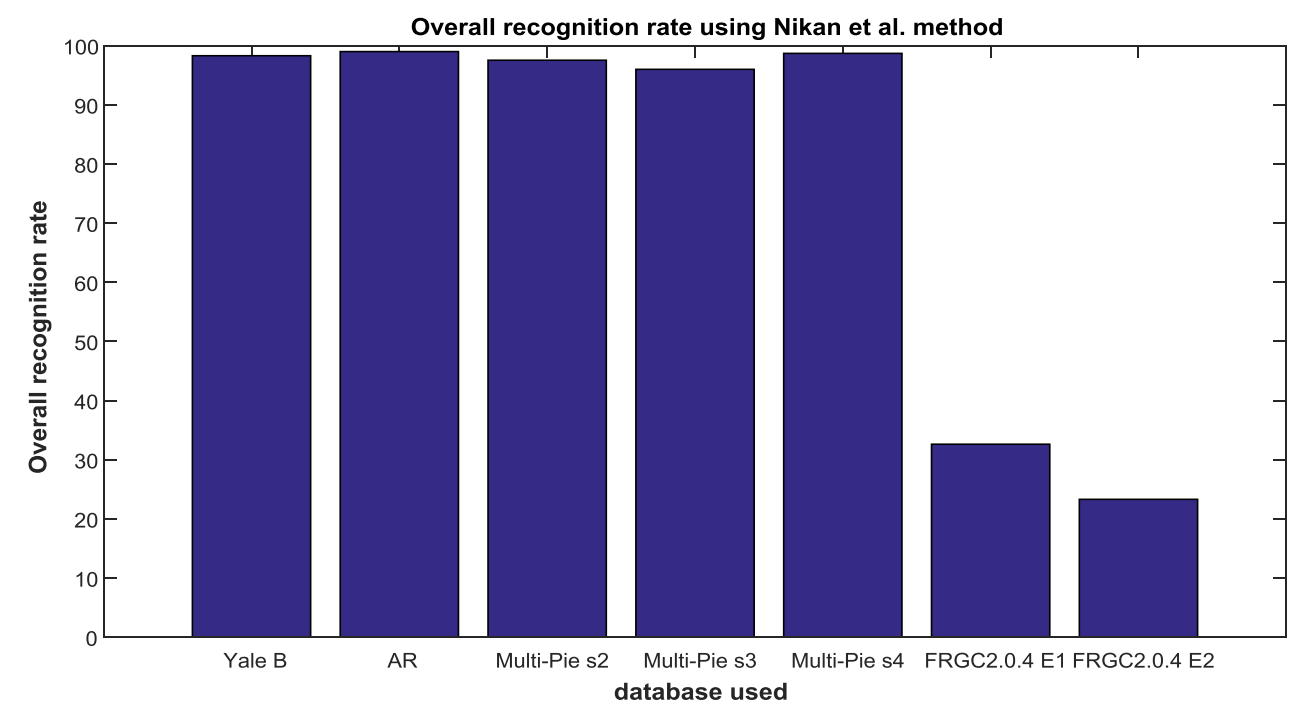

Figure 10:- Overall recognition rate using Soodeh Nikan et al. method

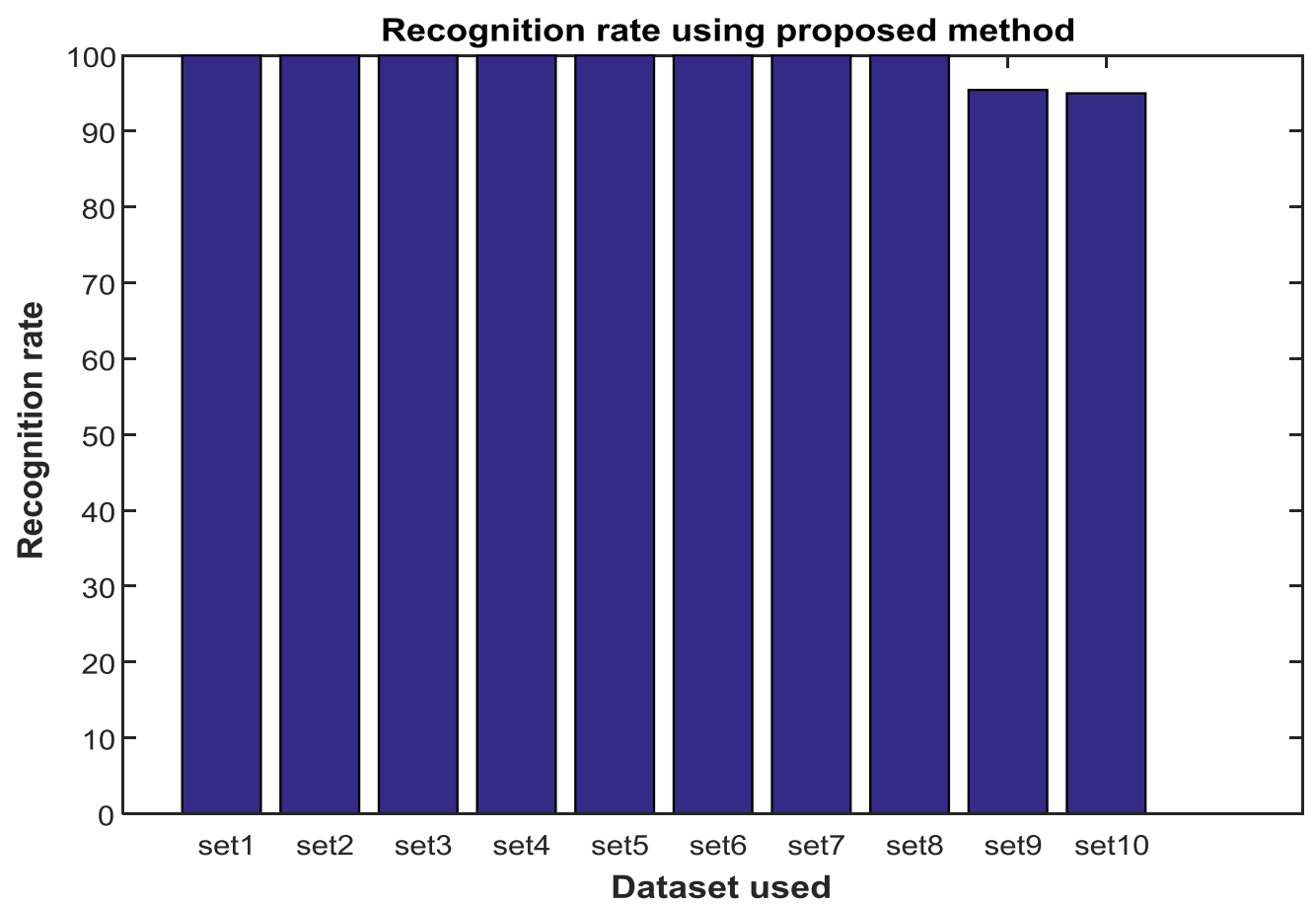

Figure 11:- Recognition rate using proposed method on individual dataset used

\begin{tabular}{|c|c|}
\hline Overall accuracy & $\mathbf{9 9 . 5} \%$ \\
\hline
\end{tabular}

Table (c) :- Approximate overall Classifier Accuracy

\section{CONCLUSION}

In this work we proposed neural network based classifier to distinguish persons having taken images in illumination variable conditions. First of all, a database has been collected of ten different persons with varying illumination conditions. In this LBP and LPQ descriptors are used to get the feature set for further classification..Therefore both these features can easily extract features of a person's face in varying illumination conditions. After this GMM is used for feature space reduction of LBP and LPQ features

\section{Future Scope}

In future algorithm can be modified or applied on different datasets especially containing human gestures like anger, fear, surprise, happiness, sad etc. Along with this, this algorithm can be used along with other biometric systems i.e. fingerprint, voice, signature etc. in order to increase the security of biometric systems. 


\section{REFRENCES}

[1] Chi Ho Chan, Muhammad Atif Tahir, Josef Kittler and Matti Pietika inen, "Multiscale Local Phase Quantization for Robust Component-Based Face Recognition Using Kernel Fusion of Multiple Descriptors." Published in ieee transactions on pattern analysis and machine intelligence, vol. 35, no. 5, may 2013.

[2] D. A. Reynolds, "Gaussian Mixture Models", In proc. Of Encyclopedia of Biometrics, pp. 659-663, 2009.

[3] Dass M. Venkat,Ali Mohammed Rahmath,Ali Mohammed Mahmood, -Image Retrieval Using Interactive Genetic Algorithm, International Conference on Computational Science and Computational Intelligence, Las Vegas, NV, Vol. 1, pp.: 215-220, May 2014.

[4] Dhall, A., Asthana, A., Goecke, R., Gedeon, T.: 'Emotion recognition using PHOG and LPQ features'. Proc. IEEE Int. Conf. on Automatic Face and Gesture Recognition and Workshops (FG11), Santa Barbara, CA, 2011, pp. 878-883

[5] EsaRahtu, JanneHeikkila, Ville Ojansivu, TimoAhonen, "Local phase quantization for blurinsensitive image analysis." Published in Image and Vision Computing archive Volume 30 Issue 8, August, 2012 Pages 501-512.

[6] Gurpreetkaur, GurpinderKaur (2012)"Classification of Biological Species Based on Leaf Architecture" International Journal of Engineering Research and Development ,ISSN: 2278-067X, Volume 1, Issue 6 (June 2012), PP.35-42

[7] Haddadnia, J.,Faez, K. and Moallem, P. "Neural Network Based Face Recognition with Moment Invariants," Proceedings of the IEEE, pp. 1018-1021, 2001.

[8] Heusch, G., Rodriguez, Y. and Marcel, S. "Local Binary Patterns as an Image Preprocessing for Face Authentication, "Proceedings of the Seventh International Conference on Automatic Faceand Gesture Recognition, pp. 6-14, April 2006.

[9] Ho Jan-Ming,LinShu-Yu,Fann Chi-Wen,Wang YuChun,Chang Ray-I, - A Novel Content Based Image Retrieval System Using K-Means With Feature Extraction,\| International Conference on Systems and Informatics, Yantai, pp.:785-790, May 2012.

[10] Iván González-Díaz, Member, IEEE, Carlos E. BazHormigos, and Fernando Díaz-de-María, Member, IEEE, "A Generative Model for Concurrent Image Retrieval and ROI Segmentation,\| IEEE Transactions on Multimedia, ISSN: 1520-9210, Issue No. 1, Vol. 16, pp.: 169-183, January 2014.

[11]Jain Arun, Aggarwalsona, “ Multimodal Biometric System: A Survey,\| International Journal of Applied Science and Advance Technology, ISSN: 0973-7405, Vol. 1, pp.: 58-63, January-June 2012.

[12] JavadHaddadnia, MajidAhmadi, and KaamranRaahemfa, "An Effective Feature Extraction Method for Face Recognition”, International Conference on Image Processing, pp. 917-920, 2003.
[13] Jeffrey R. Paone, Patrick J. Flynn, Fellow, IEEE, P. Jonathon Philips, Fellow, IEEE, Kevin W. Bowyer, Fellow, IEEE, Richard W. VorderBruegge, Patrick J. Grother,George W. Quinn, Matthew T. Pruitt, and Jason M. Grant," Double Trouble: Differentiating Identical Twins by Face Recognition." IEEE TRANSACTIONS ON INFORMATION FORENSICS AND SECURITY, VOL. 9, NO. 2, FEBRUARY 2014

[14] K. Jain, A. Ross, S. Prabhakar, "An Introduction to Biometric Recognition", IEEE Trans. on Circuits and Systems for Video Technology,Vol. 14, No. 1, pp 4-19, January 2004.

[15] KresimirDelacMislavGrgic (2004), "A Survey Of Biometric Recognition Methods", 46th International SyrnPoSium Electronics in Marine. ELMAR-2004. 1618 June 2004. Zadar. Croatia.

[16] Soodeh Nikan ,MajidAhmadi(2014),' Local Gradient Based illumination invariant Face recognition using Local Phase Quantisation and Multi Resolution local Binary Pattern Fusion ''Institution of engineering and Technology ISSN 1751-9659 vol 9,iss 1 pp 12-21 17 MAY -7 JUNE 2014

\section{BIOGRAPHIES}

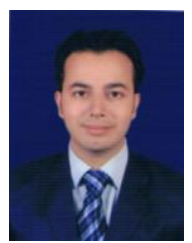

Jatinder Sharma

M.Tech Student of Bhai Gurdas Institute of Engineering and Technology

Department Electronics and communication engineering

City : Patiala-147001 (Punjab)

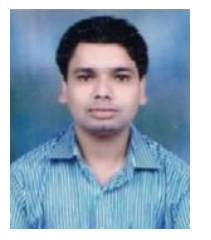

\section{Rishav Dewan}

Assistant Professeor at Bhai Gurdas Institute of Engineering and Technology

Department Electronics and communication engineering

City Patiala-147001(Punjab) 